\title{
Fitting B/C cosmic-ray data in the AMS-02 era
}

\section{Derome*}

LPSC, Université Grenoble Alpes, CNRS/IN2P3, 53 avenue des Martyrs, 38026 Grenoble, France

E-mail: laurent.derome@lpsc.in2p3.fr

\section{Maurin}

LPSC, Université Grenoble Alpes, CNRS/IN2P3, 53 avenue des Martyrs, 38026 Grenoble, France

E-mail: david.maurinelpsc.in2p3.fr

\section{P. Salati}

LAPTh, Université Savoie Mont Blanc \& CNRS, 74941 Annecy Cedex, France

E-mail: pierre.salati@lapth.cnrs.fr

\section{Boudaud}

LPTHE, Sorbonne Université \& CNRS, 4 Place Jussieu, 75252 Paris Cedex 05, France

E-mail: boudaudelpthe.jussieu.fr

\section{Y. Génolini}

Service de Physique Théorique, Université Libre de Bruxelles, Boulevard du Triomphe, CP225,

1050 Brussels, Belgium

E-mail: yoann.genolini@ulb.ac.be

\section{P. Kunzé}

LPSC, Université Grenoble Alpes, CNRS/IN2P3, 53 avenue des Martyrs, 38026 Grenoble,

France

\begin{abstract}
AMS-02 on the International Space Station has been releasing data of unprecedented accuracy. This poses new challenges for their interpretation. In this paper, we refine the methodology to get a statistically sound determination of the cosmic-ray propagation parameters. For this purpose, we inspect the impact of nuclear cross-section uncertainties and energy correlations in data systematic errors.
\end{abstract}

36th International Cosmic Ray Conference -ICRC2019-

July 24th - August 1st, 2019

Madison, WI, U.S.A.

${ }^{*}$ Speaker. 


\section{Introduction}

Particle physics detectors in space have opened a new era for the study of Galactic cosmic rays (GCRs). The Alpha Magnetic Spectrometer (AMS-02) instrument on the International Space Station (ISS) provides the best data to date for leptons and nuclei [1-8], with an uncertainty of a few percent on a large energy range. Promises of high-precision cosmic-ray (CR) physics can only be fulfilled if the various sources of uncertainties, model and data, are fully accounted for.

The dominant source of uncertainty in the modelling is from nuclear cross sections $[9,10]$. In this study, mock data are used to characterise the bias on reconstructed model parameters and to assess how well nuisance parameters on cross sections allow one to recover unbiased values of these parameters.

AMS-02 systematic uncertainties dominate the error budget of measured fluxes and ratios overall. In experiments measuring spectra, correlations in adjacent energy bins may be introduced at the data analysis stage. Such a correlation matrix is not available; however, we can rely on educated guesses to derive it and inspect the consequences on the model parameters.

\section{Model and parameters}

For simplicity, and because most of our results and conclusions should not depend on this modelling, we use throughout the paper a 1D diffusion model, as implemented in the USINE package [11]. We rely on two configurations, which correspond to two extreme cases of a more generic parametrisation of the diffusion coefficient.

Model A is a diffusion-convection-reacceleration model with $K(R)=\beta^{\eta_{t}} K_{0}\left(\frac{R}{1 \mathrm{GV}}\right)^{\delta} \times K_{\mathrm{HE}}(R)$ where $\eta_{t}$ allows for a sub-relativistic change of the diffusion coefficient as parametrised in [10], and where $K_{H E}(R)=\left(1+\left(R / R_{h}\right)^{\delta_{h} / s_{h}}\right)^{-s_{h}}$ is a high energy break whose parameter values are taken from [12]. Following [13], the diffusion coefficient in momentum is taken as $K_{p p}(R) \times K(R)=$ $\frac{4\left(V_{a} \beta E\right)^{2}}{3 \delta\left(4-\delta^{2}\right)(4-\delta)}$. This configuration has 5 free transport parameters: $K_{0}, \delta, \eta_{t}, V_{a}$, and $V_{c}$.

Model B is a pure diffusion model (no $V_{c}$, no $V_{a}$ ) with a double broken power-law, at both high and low energy: $K(R)=\beta K_{0}\left(\frac{R}{1 \mathrm{GV}}\right)^{\delta}\left(1+\left(\frac{R_{l}}{R}\right)^{\left(\delta+\delta_{l}\right) / s_{l}}\right)^{s_{l}} \times K_{H E}(R)$. This configuration has 4 free parameters: $K_{0}, \delta, R_{l}$, and $\delta_{l}$. The smoothness parameter $s_{l}$ has only a minor impact on the results, so it is fixed to 0.05 (quick transition) to speed up the fitting procedure.

\section{Handling cross-section uncertainties}

Nuclear cross sections are measured by 'external' experiments, and these measurements can be incorporated as a distribution of probability in the $\chi^{2}$ minimisation via nuisance parameters: cross sections far from their most probable values must be penalised in the minimisation.

The difficulty lies in the characterisation of the uncertainties, the choice of the nuisance parameters, and assessing the robustness of the procedure. We start by characterising the impact of crosssection uncertainties on the $\mathrm{B} / \mathrm{C}$ ratio. Cross section data uncertainties are typically at $\sim 5-10 \%$ level for inelastic cross sections, and $15-25 \%$ level for production cross sections [14]. However, because the data are sometimes scarce, old, not always consistent with one another, and sometimes 
even missing for some reactions, several parametrisation of the whole network of reactions exist. A conservative estimate of the impact of cross-section uncertainties on the $\mathrm{B} / \mathrm{C}$ calculation can be based on the scatter observed from using several of these parametrisations (see [14] for more details). For inelastic cross sections, $\sigma_{\text {inel }}$, we use below B94 [15], W96 [16], T99 [17, 18], and W03 [19]. Except for T99, the scaling $\sigma_{\mathrm{He}} / \sigma_{\mathrm{H}}$ is taken from [20]. For production cross sections, $\sigma_{\text {prod }}$, we use W98 [21-23], S01 ${ }^{1}$, W03 [19], and G17 [24, 25].

For a given set of propagation parameters, we calculate the $\mathrm{B} / \mathrm{C}$ ratio for various parametrisations, and we plot in Fig. 1 the relative variation with respect to a reference (T99 for $\sigma_{\text {inel }}$ and W03 for $\sigma_{\text {prod }}$ ). The maximum impact of inelastic cross sections is $\lesssim 3$ at $\sim 5 \mathrm{GV}$ (left panel). It slowly decreases to zero at higher $R$, because the escape time from the Galaxy decreases with $R$ while the destruction time remains constant. The maximum impact of the production cross sections is $\lesssim 10 \%$, and it is equally seen at low and high rigidity (right panel) because the Boron flux is directly related to its production cross section.

To choose nuisance parameters so that they enable to move from one parametrisation to another, one can start from a reference cross section and apply several simple transformations on the normalisation, on the energy scale, or on the slope below $E_{k / n}^{\text {thresh }}$. This set of transformations is denoted NSS in the following. For each reaction, the NSS nuisance parameters are chosen so that $\sigma^{\mathrm{NSS}} / \sigma^{\text {ref }} \pm 1 \sigma$-calculated from Gaussian distributed samples of $(\mu, \sigma)^{\text {Norm, Scale, Slope }}$ encompasses the various cross-section parametrisations. A second and more straightforward option is to define cross sections as a linear combination of the available cross-section parametrisations. The $C_{i}$ parameters are taken to be flat in $[-0.5,1.5]$ and are forbidden to wander outside this range. 1000 mock data, based on given values of the transport parameters, and a given choice of cross-section parametrisations are generated. The $\chi^{2}$ analysis ${ }^{2}$ is done fitting mock data with cross sections that are the same, that is unbiased case, or differ from the ones used to generate them, that is biased case. We show in Fig. 2, from top to bottom, the $\chi_{\min }^{2} /$ dof distribution and the 1D probability distribution function of all transport parameters. For readability, we only show the results for the No nuis. (black lines) and with nuis. Inel.+Prod. (green lines) cases. In this figure, solid (dashed) lines correspond to NSS (LC) nuisance type.

Impact on goodness of fit (top panels): the black lines, which correspond to a fit with the transport parameters only (No nuis.), show that using wrong cross sections can lead to $\chi_{\min }^{2} /$ dof values larger than one. Taken at face value, one would conclude that the model is excluded. Adding crosssection nuisance parameters-which encompass the true cross-section values at $1 \sigma$-allows to recover $\chi_{\min }^{2} /$ dof $\sim 1$ (green lines). The LC nuisance parameters (green dashed lines) fare slightly better than NSS ones (green solid lines): this is understood as the 'true' cross-section values can be reached in the LC case, whereas they can only be approached in the NSS case.

Biased transport parameters (remaining panels): without nuisance parameters (black lines), the transport parameters are strongly biased, up to several $\sigma$ away from their true value (vertical dashed line). Using nuisance parameters (black vs green lines) has two effects: it enlarges the probability distribution function of the transport parameters, and it shifts the distribution towards the true value. Overall, the two schemes allow to recover unbiased parameters. A mismatch is

\footnotetext{
${ }^{1}$ Same dataset as in [19], but fitted by Aimé Soutoul (private communication).

${ }^{2}$ Statistical uncertainties only (taken from real data) are used in our analysis, in order to disentangle the issue of cross-section uncertainties and more involved data uncertainties (discussed in Sect. 4).
} 
observed for the strongly correlated $\delta$ and $K_{0}$ parameters when using NSS in Model B. The latter is particularly sensitive to any small energy-dependent difference in the cross-section values as it directly reflects on the calculated B/C. On the other hand, in Model A, this difference can be smoothed out by reacceleration.

We finally comment on the fact that the LC case does not recover fully unbiased transport parameters. Whereas nuisance parameters enable the cross sections to match their 'true' values (the one used to generate the data), they can only do so for the selected four production cross sections and three inelastic reactions. The remaining ones are different from those used to generate the mock. This 'reaction network' effect explains the observed residual biases.

We see that assuming wrong cross sections can strongly bias the model fit, and thus bias the deduced transport parameters. Starting from the wrong cross-section values, we show that nuisance parameters on a limited number of reactions allow to mostly recover the true values of the transport parameters. However, the procedure is not perfect owing to 'reaction network' effects, that is the fact that we only use as nuisance a small, though representative, sample of all the reactions involved. The LC parameters fare slightly better than NSS parameters, but this is only true because LC parameters always contain 'true' cross sections of the analysis. In real life, we do not know what are the real cross sections, and there is no guarantee that the LC approach would still fare better than the NSS one.

\section{Handling systematics from experimental data}

Almost all, if not all CR phenomenological studies, account for data uncertainties as the quadratic sum of statistical and systematics uncertainties. Doing so ignores any possible energy correlations for the systematic errors. This has two important consequences on the model bestfit analysis. For instance, considering two extreme cases, fully uncorrelated and fully correlated uncertainties, corresponds to adding quadratically the uncertainties or to allow for a global normalisation of the data (or more precisely to an energy-dependent normalisation related to the energy dependence of the uncertainty). Starting from the same uncertainties, a $\chi^{2}$ analysis on the two different cases would lead to a smaller $\chi_{\min }^{2}$ in the former than in the latter case, and possibly to different values for the best-fit parameters of the model. A better approach is to use the correlation matrix of error in the $\chi^{2}$ analysis. However, the AMS-02 collaboration does not provide this matrix, and we have to rely on the provided information to build one. We then inspect how sensitive the analysis is on our choices.

The errors on the B/C ratio measured by AMS-02 are described in [7]. The different contributions obtained from table VI of the Supplemental Material of [7] are shown in Fig. 3 as thick solid lines. As explained in [7], the unfolding error (Unf.) corresponds to the contribution coming from the uncertainty on the rigidity resolution function and the unfolding procedure. The rigidity scale error (Scale) is the sum of the contribution from residual tracker misalignment and from the uncertainty on the magnetic field map measurement and its temperature time-dependent correction. The acceptance error (Acc.) is the sum of different contributions: survival probability of Boron and Carbon in the detector, Boron contamination from heavier nuclei fragmentation (mainly carbon), and uncertainty on the 'data/Monte Carlo' corrections to the Boron and Carbon acceptances. 

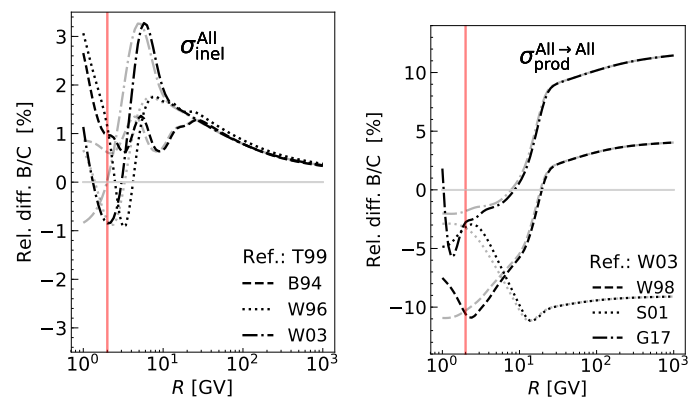

Figure 1: Impact of different cross-section parametrisations on $\mathrm{B} / \mathrm{C}$ flux calculation as a function of rigidity, w.r.t. a reference (denoted Ref. in the legend). The left and right panels are for inelastic and production cross sections respectively. The thick (resp. thin) lines are for the interstellar (resp. solar-modulated at $\phi_{\text {Force-Field }}=800 \mathrm{MV}$ ) calculation. The red vertical line highlights the first rigidity point of AMS- $02 \mathrm{~B} / \mathrm{C}$ data.
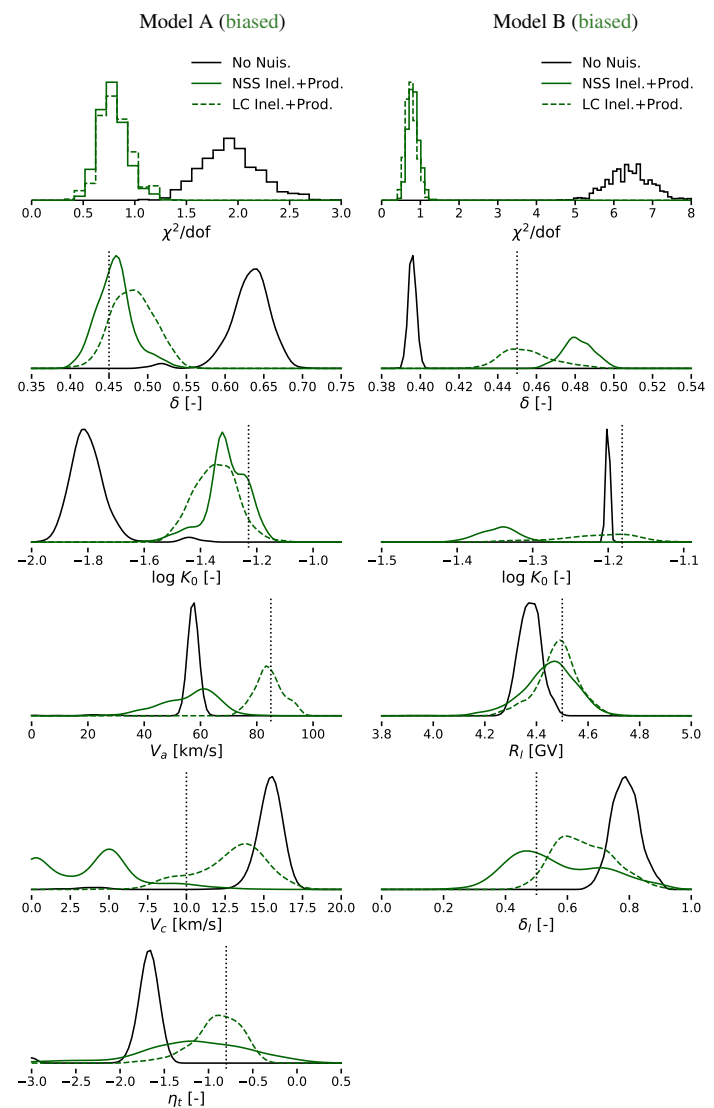

Figure 2: Distribution of best-fit values $\left(\chi_{\min }^{2}\right.$, then transport parameters) from the analysis of 1000 mock data for Model A (left panel) and B (right panel), for the biased case. The line style and colours indicate the type and configuration of nuisance parameters. The vertical dashed lines represent true values.

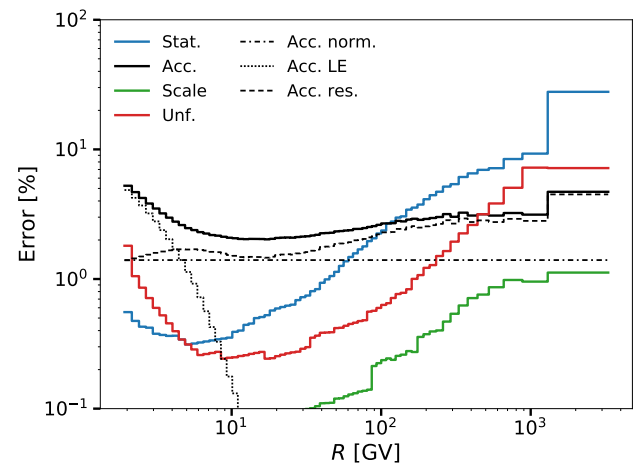

Figure 3: AMS- 02 errors for $\mathrm{B} / \mathrm{C}$ data. Solid lines correspond to the errors provided in [7], namely statistical, acceptance, scale, and unfolding (the step-like evolution is artificial and related to the rounding of the values provided in the table). The thin blacks lines correspond to a further split of the acceptance errors: normalisation (norm.), low energy (LE), and residual (res.).

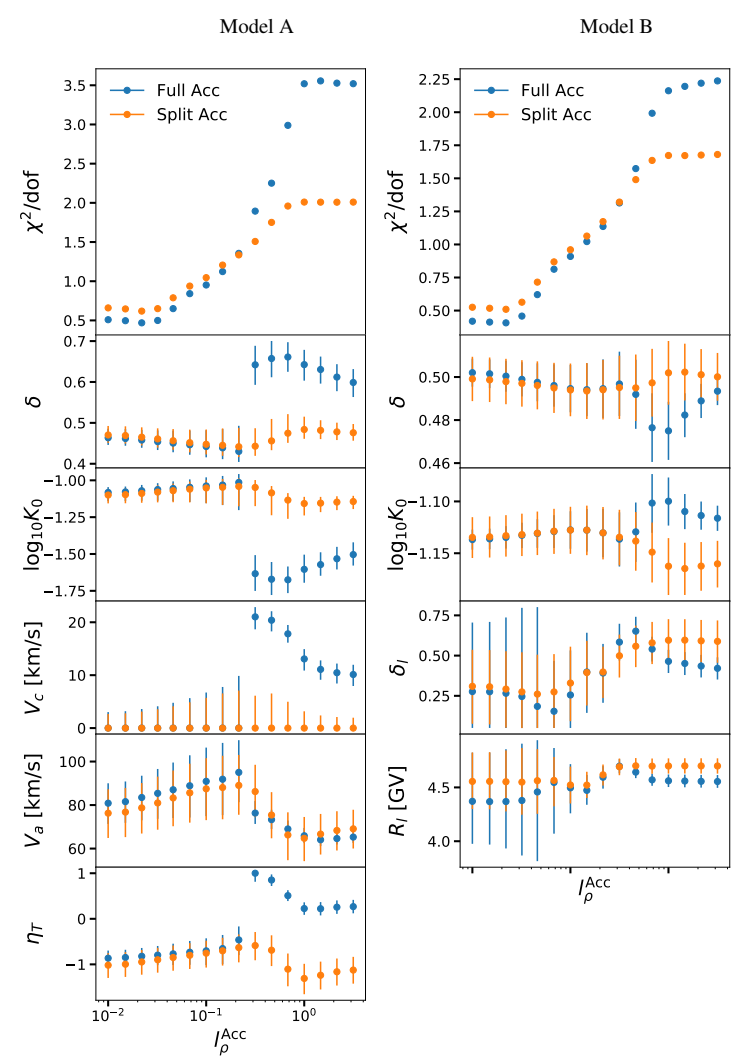

Figure 4: Values obtained for the $\chi_{\min }^{2} /$ dof and the fit parameters (and uncertainties) as a function of $l_{\rho}^{\text {Acc. for }}$ propagation models A (left) and B (right) and for the full acceptance error (blue) and the split acceptance error (orange). In the case of split acceptance error, $l_{\rho}^{\text {Acc. }}$ corresponds to the correlation length of the Acc. res. contribution only. 
To properly take into account AMS-02 data uncertainties, one needs to define the covariance matrices $C_{\alpha}$ for $\alpha=$ (Stat., Unf., Scale, Acc.), and minimise the $\chi^{2}$. As these covariance matrices are not provided explicitly in [7], we start from the covariance matrices of relative errors $C_{\text {rel }}^{\alpha}$, estimated from the following expression:

$$
\left(C_{\text {rel }}^{\alpha}\right)_{i j}=\sigma_{i}^{\alpha} \sigma_{j}^{\alpha} \exp \left(-\frac{1}{2} \frac{\left(\log \left(R_{i} / R_{j}\right)^{2}\right.}{\left(l_{\rho}^{\alpha}\right)^{2}}\right),
$$

with $\left(C_{\mathrm{rel}}^{\alpha}\right)_{i j}$ the $i j$-th element built from the relative errors $\sigma_{i}^{\alpha}$ and $\sigma_{j}^{\alpha}$ at rigidity bins $R_{i}$ and $R_{j}$, and where the parameter $l_{\rho}^{\alpha}$ is the correlation length associated with the error $\alpha$ (in unit of decade of rigidity).

For this study, we set the correlation lengths $l_{\rho}^{\alpha}$ to the following values:

- $l_{\rho}^{\text {Stat. }}=0$ because the number of events on each bin are independent;

- $l_{\rho}^{\text {Scale }}=\infty$ since the uncertainty on the rigidity scale affects all rigidities similarly;

- $l_{\rho}^{\text {Unf. }}=0.5$ because errors from the unfolding procedure and from the rigidity response function affect intermediate scales. As seen on Fig. 3, this error is sub-dominant compared to Stat. and Acc. errors, and we checked that the results are not affected by our choice for $l_{\rho}^{\text {Unf.; }}$

- The value of the correlation length for the Acc. error is more critical, because this error dominates the systematic error and it cannot be easily defined. The dependence of $\chi_{\min }^{2} /$ dof and of the fitted parameters with this correlation is studied below for different values $l_{\rho}^{\text {Acc. }}=$ $0.01 \ldots 3$, which cover the range from lower than the bin size (fully uncorrelated) to the full range (fully correlated).

As the acceptance error is a combination of errors which are expected to have a rather small correlation length ('data/Monte Carlo' corrections) and others which are expected to have a large correlation length (cross-section normalisation), one can try to decompose this error into different contributions with different correlation lengths. In particular, the rise of the acceptance error at low rigidity is not expected to be correlated with larger rigidities: it is related to the rapid change of the acceptance at low energy mostly because of energy losses in the detector. One can therefore construct a better description of the covariance matrix by splitting acceptance errors in three independent parts:

- a normalisation error, Acc.norm. (dash-dotted dark line in Fig 3), with a large correlation length $\left(l_{\rho} \sim 1.0\right)$;

- a rise at low rigidity, Acc. $L E$ (dark line), with an intermediate correlation length $\left(l_{\rho} \sim 0.3\right)$;

- a residual error, Acc.res. (dashed dark line), defined so that the quadratic sum of the three contributions equals the full acceptance error. This last part corresponds mainly to 'data/Monte Carlo' corrections and the rigidity-dependent parts of other acceptance errors. Its correlation length is not well defined and left free in the following. 
The Fig. 4 shows the values obtained for $\chi_{\min }^{2} /$ dof and best-fit parameters as a function of $l_{\rho}^{\text {Acc. }}$ for models A (left) and B (right) and for the full acceptance error (blue circles) and the split

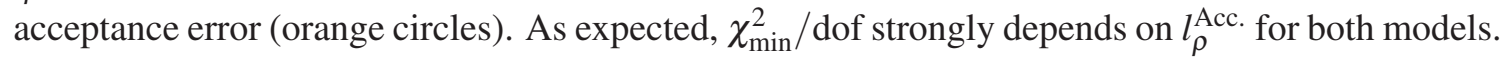
The best-fit parameters are stable (i.e. fluctuate within errors estimated from the fit) for low and large $l_{\rho}^{\text {Acc. }}$ but undergo a rapid jump around $l_{\rho}^{\text {Acc. }}=2$ for model A when one uses the full acceptance error description. These features are problematic since it means that the best-fit parameters are very sensitive to the choice of $l_{\rho}^{\text {Acc. }}$. In addition, with the full acceptance error, the best-fit obtained for model A and $l_{\rho}^{\text {Acc. }} \approx 1$ does not pass through the data points.

From the above results, we conclude that the best way to handle the systematic errors is to use the split acceptance errors approach. Indeed, not only does it provide a more realistic description of the acceptance systematic error, but it also leads to more stable results w.r.t. the values taken for $l_{\rho}^{\text {Acc. }}$. In this approach, $l_{\rho}^{\text {Acc. }}=0.1$ is a reasonable choice which gives a $\chi_{\min }^{2} /$ dof $\sim 1$ and conservative errors for the fit parameters.

\section{Conclusions}

Faced with the challenges of interpreting cosmic-ray data of unprecedented accuracy, we have refined the methodology to properly account for uncertainties in the fit to the data. The proposed methodology was exemplified on the analysis of the AMS- $02 \mathrm{~B} / \mathrm{C}$ ratio.

The first step was to handle properly cross-section uncertainties. We proposed an approach to account for these uncertainties, based on a combination of Normalisation, Scale, and lowenergy Slope cross-section modifications (NSS) or based on linear combinations (LC) of existing parametrisations. We validated this choice on simulated data, showing that the degrees of freedom enabled by these nuisance parameters allow to recover the true parameters (when starting from a different set of cross sections simulated data were generated with).

The second step was to handle as best as possible data uncertainties. We accounted for possible energy correlations in the AMS- 02 data via the covariance matrix of errors. The crucial parameters are the correlation length associated with various systematics, correlating more or less strongly various energy bins. These correlations not only possibly biases the transport parameters fit to the data, but it also has a huge impact on the statistical interpretation of the model inspected.

The methologgy presented here have been used to establish new benchmark scenarios based on fits to the AMS-02 boron to carbon ratio [26].

\section{References}

[1] M. Aguilar, G. Alberti, B. Alpat, A. Alvino, G. Ambrosi, K. Andeen, H. Anderhub, L. Arruda, P. Azzarello, A. Bachlechner, and et al. Phys. Rev. Lett., 110(14):141102, April 2013.

[2] M. Aguilar, D. Aisa, B. Alpat, A. Alvino, G. Ambrosi, K. Andeen, L. Arruda, N. Attig, P. Azzarello, A. Bachlechner, and et al. Phys. Rev. Lett., 113(22):221102, November 2014.

[3] M. Aguilar, D. Aisa, A. Alvino, G. Ambrosi, K. Andeen, L. Arruda, N. Attig, P. Azzarello, A. Bachlechner, F. Barao, and et al. Phys. Rev. Lett., 113(12):121102, September 2014.

[4] M. Aguilar, D. Aisa, B. Alpat, A. Alvino, G. Ambrosi, K. Andeen, L. Arruda, N. Attig, P. Azzarello, A. Bachlechner, and et al. Phys. Rev. Lett., 114(17):171103, May 2015 a. 
[5] M. Aguilar, D. Aisa, B. Alpat, A. Alvino, G. Ambrosi, K. Andeen, L. Arruda, N. Attig, P. Azzarello, A. Bachlechner, and et al. Phys. Rev. Lett., 115(21):211101, November 2015b.

[6] M. Aguilar, L. Ali Cavasonza, G. Ambrosi, L. Arruda, N. Attig, S. Aupetit, P. Azzarello, A. Bachlechner, F. Barao, A. Barrau, and et al. Phys. Rev. Lett., 117(23):231102, December 2016.

[7] M. Aguilar, L. Ali Cavasonza, G. Ambrosi, L. Arruda, N. Attig, S. Aupetit, P. Azzarello, A. Bachlechner, F. Barao, A. Barrau, and et al. Phys. Rev. Lett., 120(2):021101, January 2018.

[8] M. Aguilar, L. Ali Cavasonza, B. Alpat, G. Ambrosi, L. Arruda, N. Attig, S. Aupetit, P. Azzarello, A. Bachlechner, F. Barao, and et al. Phys. Rev. Lett., 121(5):051103, August 2018.

[9] F. Donato, D. Maurin, P. Salati, A. Barrau, G. Boudoul, and R. Taillet. ApJ, 563:172-184, December 2001.

[10] D. Maurin, A. Putze, and L. Derome. A\&A, 516:A67, June 2010.

[11] D. Maurin. ArXiv e-prints 1807.02968, July 2018.

[12] Y. Génolini, P. D. Serpico, M. Boudaud, S. Caroff, V. Poulin, L. Derome, J. Lavalle, D. Maurin, V. Poireau, S. Rosier, P. Salati, and M. Vecchi. Phys. Rev. Lett., 119(24):241101, December 2017.

[13] E. S. Seo and V. S. Ptuskin. ApJ, 431:705-714, August 1994.

[14] Y. Génolini, D. Maurin, I. V. Moskalenko, and M. Unger. Phys. Rev. C, 98(3):034611, September 2018.

[15] V. S. Barashenkov and A. Polanski. Technical Report E2-94-417, Comm. JINR, Dubna, 11994.

[16] H. P. Wellisch and D. Axen. Phys. Rev. C, 54:1329-1332, September 1996.

[17] R. K. Tripathi, F. A. Cucinotta, and J. W. Wilson. Nucl. Instrum. Methods Phys. Res. B, 117:347-349, October 1996.

[18] R. K. Tripathi, F. A. Cucinotta, and J. W. Wilson. Nucl. Instrum. Methods Phys. Res. B, 155:349-356, September 1999.

[19] W. R. Webber, A. Soutoul, J. C. Kish, and J. M. Rockstroh. ApJS, 144:153-167, January 2003.

[20] P. Ferrando, W. R. Webber, P. Goret, J. C. Kish, D. A. Schrier, A. Soutoul, and O. Testard. Phys. Rev. C, 37:1490-1501, April 1988.

[21] W. R. Webber, J. C. Kish, J. M. Rockstroh, Y. Cassagnou, R. Legrain, A. Soutoul, O. Testard, and C. Tull. ApJ, 508:940-948, December 1998.

[22] W. R. Webber, J. C. Kish, J. M. Rockstroh, Y. Cassagnou, R. Legrain, A. Soutoul, O. Testard, and C. Tull. ApJ, 508:949-958, December 1998.

[23] W. R. Webber, A. Soutoul, J. C. Kish, J. M. Rockstroh, Y. Cassagnou, R. Legrain, and O. Testard. Phys. Rev. C, 58:3539-3552, December 1998.

[24] I. V. Moskalenko, S. G. Mashnik, and A. W. Strong. ICRC, 5:1836-1839, August 2001.

[25] I. V. Moskalenko and S. G. Mashnik. ICRC, 4:1969, July 2003.

[26] Y. Génolini, M. Boudaud, P.-I. Batista, S. Caroff, L. Derome, J. Lavalle, A. Marcowith, D. Maurin, V. Poireau, V. Poulin, S. Rosier, P. Salati, P. D. Serpico, and M. Vecchi. Phys. Rev. D, 99:123028, Jun 2019. 